\title{
11 Glossary of terms and key assessment methods
}

In the present EURACT Performance Agenda, a lot of educational terms are used. In the addendum readers can find a short glossary on some of these educational terms, predominantly taken from the EURACT Educational Agenda. Description of types of assessment is partially based on Newble and Cannon ${ }^{105}$. Key assessment methods presented in the Agenda are described in the last paragraph (terminology on assessment tools and methods). Arrows $(\rightarrow$ ) refer to other terms in the glossary.

\subsection{More general terminology}

Basic medical education, BME

The part of the medical $\rightarrow$ curriculum that relates to all medical students, to give a sound basis for further $\rightarrow$ vocational or specialty training. The European Union claims a minimum of six years of BME.

\section{Bachelor degree}

Following the Bologna Declaration 1999 to create a "European Higher Education Area" by 2010 , the first basic part in each $\rightarrow$ curriculum should preferable have 3 years and 180 study points.

\section{Continuing medical education, CME}

Any and all ways by which a graduated physician continues to learn and change in practice in a lifelong learning scheme.

\section{Continuing professional development, CPD}

A process of planned and individually tailored learning in practice with a focus on the quality of care. CPD includes the identification of learning needs, construction of a learning agenda, drawing a concrete learning plan, and controlling this in an educational $\rightarrow$ portfolio format. As it relates to lifelong learning, it can become a lifelong Personal Development Plan ${ }^{106}$.

\section{Convergence}

Voluntary adoption of suitable policies for the achievement of a common goal.

\section{Credit}

The "currency" used to measure students' $\rightarrow$ workload in terms of the notional learning time required to achieve specified $\rightarrow$ learning outcomes. 


\section{Credit framework}

A system that facilitates the measurement and comparison of $\rightarrow$ learning outcomes achieved in the context of different qualifications, programmes of study and learning environments.

\section{Credit level}

An indicator of the relative demand of learning and of $\rightarrow$ learner autonomy. It can be based on the year of study and/or on course content (e.g. Basic, Intermediate, Advanced, and Specialized).

\section{Credit type}

An indicator of the status of course units in the programme of study. It can be described as Core (major course unit), Related (unit providing instruments/support) and Minor (optional course unit).

\section{Curriculum}

In formal education, a curriculum is the planned interaction of $\rightarrow$ learners with instructional content, materials, resources, and processes for evaluating the attainment of educational objectives. The general practice curriculum in $\rightarrow$ vocational training defines the learning outcomes for the specialty of general practice and delivers a full description of the $\rightarrow$ knowledge, skills, attitudes and behaviours required of a GP in managing patients and their problems.

\section{Doctorate or Doctoral degree}

A high level qualification which is internationally recognized as qualifying someone for research or academic work. It will include a substantial amount of original research work which is presented in a thesis. It is generally referred to as the degree awarded after completion of third cycle studies.

\section{ECTS (European Credit Transfer System)}

A system for increasing the transparency of educational systems and facilitating the mobility of students across Europe through $\rightarrow$ credit transfer. It is based on the general assumption that the global $\rightarrow$ workload of an academic year of study is equal to 60 credits. The 60 credits are then allocated to course units to describe the proportion of the learners' workload required to achieve the related $\rightarrow$ learning outcomes. Credit transfer is guaranteed by explicit agreements between the home institution, the host institution and the mobile $\rightarrow$ learner. 


\section{First degree qualification}

First higher education qualification taken by the $\rightarrow$ learner. It is awarded after successful completion of first cycle studies which, according to the Bologna Declaration should normally last a minimum of three years or $180 \rightarrow$ ECTS credits.

\section{(Initial) Master degree}

Following the Bologna Declaration 1999 to create a "European Higher Education Area" by 2010, after obtaining first a bachelor degree, a second part in the $\rightarrow$ curriculum leads to a master degree. It should have a minimum of 2 years and $120 \rightarrow$ ECTS-study points. For medical master studies, a clinical period of 3 to 4 years is accepted in many European countries. Obtaining a master degree normally includes some form of master thesis.

\section{Master after Master or postinitial Master degree}

Following the Bologna Declaration 1999 to create a "European Higher Education Area" by 2010, after obtaining an $\rightarrow$ initial master, programmes can give entrance to a postinitial master programme. In this logic, $\rightarrow$ specialty training should be considered a postinitial master programme.

\section{Specialty training}

\section{$\rightarrow$ vocational training}

\section{Tuning}

Developing agreement and harmony by combining single sound into a common "tune" or pattern of sounds. It is used in the "tuning project" to achieve a form of harmonization by finding points of $\rightarrow$ convergence and common understanding.

\section{Vocational training, VT, syn. specialty training, ST}

The part of the medical $\rightarrow$ curriculum that comes after the common $\rightarrow$ basic medical education programme for all medical students, and focuses on the acquisition of the $\rightarrow$ competences, required for the specialty discipline and related tasks in healthcare.

\subsection{Terminology on educational content}

\section{Attachment}

A period of longer attachment in a practice setting, also called "preceptorship" or "clerkship". 


\section{Competence}

The capability to successfully perform discrete observational tasks in a defined $\rightarrow$ assessment environment, in isolation from actual work. In the Miller terminology, it includes the level of "knowing" (basic facts), "knowing how" (able to apply knowledge) and "showing how" (able to apply $\rightarrow$ knowledge) but it excludes the "doing" level, the $\rightarrow$ performance in practice.

\section{Elective course}

A $\rightarrow$ course to be chosen from a predetermined list.

\section{Knowledge}

Facts, information, and skills acquired through experience or education by perceiving, discovering, or learning; the theoretical or practical understanding of a subject. It can be implicit (as with practical skill or expertise) or explicit (as with the theoretical understanding of a subject); it can be more or less formal or systematic. Knowledge acquisition involves complex cognitive processes: perception, communication, association and reasoning.

\section{Learners}

Refers to students in $\rightarrow$ basic medical education (BME) as well as $\rightarrow$ vocational trainees (VT) or practicing doctors in $\rightarrow$ continuing medical education (CME) or their $\rightarrow$ continuing professional development (CPD) and to all those who take part in the training programmes.

\section{Learning outcomes}

Statements on what a $\rightarrow$ learner is expected to know, understand and/or be able to demonstrate after completion of a process of learning. Learning outcomes are distinct from the aims of learning, in that they are concerned with the achievements of the learner rather than the overall intentions of the $\rightarrow$ teacher. Learning outcomes must be accompanied by appropriate $\rightarrow$ assessment criteria which can be used to judge that the expected learning outcomes have been achieved. Learning outcomes together with assessment criteria specify the minimum requirements for the award of $\rightarrow$ credit, while marking is based on attainment above or below the minimum requirements for the award of credit.

\section{Mark}

Any numerical or qualitative scale used to describe the results of $\rightarrow$ assessment in an individual $\rightarrow$ course unit or module. 
Mentor

$\rightarrow$ tutor

\section{Objectivistic learning}

Traditional education model, based on $\rightarrow$ knowledge transfer from $\rightarrow$ teacher to $\rightarrow$ learner. It is highly teacher centred. Content is structured in handbooks, teaching is mainly focused on lecturing by experienced teachers, and behaviour copied from experienced role models.

\section{Performance}

The level of actual performance in clinical care and communication with patients in daily practice. It relates in the Miller terminology to the "doing" level. It is considered highly dependent on existing healthcare conditions and requirements, financial and structural opportunities, practice opportunities and support.

\section{Problem based learning}

Educational model that takes the problem of the patient and the doctor as the starting point for the learning $\rightarrow$ curriculum. It is highly $\rightarrow$ learner centred, optimizes the use of pre-existing $\rightarrow$ knowledge, and stimulates self learning and search strategies.

\section{Social constructivistic learning}

Educational model that puts the learning process of the student as the central point. Learning is seen as a process, highly dependent on pre-existing $\rightarrow$ knowledge and on learning context. $\rightarrow$ Teachers are mainly architects of the stimulating learning environment for the $\rightarrow$ learners. Individual variety in learning strategies are stimulated.

\section{Teacher}

Refers to all professionals involved in an educational event as experts.

Tutor, syn. mentor, facilitator

A professional involved in the educational process as leader of the process, to guide and reflect to the benefit of the $\rightarrow$ learner(s).

\section{Workload}

All learning activities required for the achievement of the $\rightarrow$ learning outcomes (i. e. $\rightarrow$ lectures, practical work, information retrieval, private study, etc.). 


\subsection{Terminology on learning methods}

Clinical work/clinical practice under supervision

Teaching during working in clinical environment, in general practice/family practice/primary care setting. It can be organised with or without $\rightarrow$ supervision.

\section{Courses}

Structured programme of educational content, often presented in an oral format, supported by course material.

\section{Discussion}

Discussion session on a specific topic or case presentation. Can be organised as a one to one session with $\rightarrow$ tutor or $\rightarrow$ supervisor, a peer group session, a small group session like a focus group or a Balint group, or it can be a large/temporary group session at $\rightarrow$ seminars, $\rightarrow$ lectures or $\rightarrow$ workshops.

\section{Interactive (IT based) learning}

Combination of modular $\rightarrow$ reflection packages, linking case studies, focused reflection, $\rightarrow$ discussion forums, library search and/or reflection in one educational process.

\section{Lecture}

Provision of teaching content by presentation and explanation (possibly including a demonstration) by a lecturer.

\section{Literature search}

Learning to perform a medical database search in EbM, including defining a clinical question, looking for medical evidence, critical $\rightarrow$ reflection on evidence and implementation in practice.

\section{Observation}

Learning through reflective observation by a $\rightarrow$ tutor/ $\rightarrow$ supervisor in different educational settings: sit-in with real patients or $\rightarrow$ simulated patients; learning through video-taped consultation of real patient or simulated patient (observation by oneself, tutor/supervisor, peers, etc).

\section{Reading/studying}

Reading books, protocols, EbM information, novels, narratives, internet etc. 


\section{Reflection}

On self, e. g. by using a diary, videos, or in a participative reflection group.

\section{Role playing}

Using the act of playing a role as a patient, as an accompanying person, as a doctor, as a nurse etc to derived educational insight in feelings, intentions and actions.

\section{Project work}

Working out a personal project or as part of a group in a defined format: $\rightarrow$ audit project, research project, field work project etc.

\section{Seminar}

A period of instruction based on written or oral contributions by the $\rightarrow$ learners, usually in small groups.

\section{Skills training}

Learning procedural skills in adapted specific settings like: doing procedures e.g. in a skills lab, learning consultation skills by e.g. $\rightarrow$ role playing, learning (medical) database searching, learning leadership skills by running an educational or targeted meeting etc.

\section{Study visit}

Educational visit to a practice, to clinical premises, to social-welfare institutions, to health authorities etc.

\section{Supervision/supervisor}

Supervision involves regular, ongoing structured meetings/sessions with and $\rightarrow$ feedback from personal $\rightarrow$ tutor/supervisor on various topics.

\section{Writing of patient studies, case studies}

Educational activity, with a given task to provide a written description and/or $\rightarrow$ reflection document, to get $\rightarrow$ feedback from a $\rightarrow$ tutor $/ \rightarrow$ supervisor.

\section{Workshop}

A supervised session where $\rightarrow$ learners work on individual tasks and receive assistance and direction when needed. 


\subsection{Terminology on assessment tools and methods}

\section{Appraisal}

A structured process of facilitated self $\rightarrow$ reflection $^{107}$ and a means of aiding GP personal development. Key roles may include annual exploration of role expectations; a review of progress towards previously agreed objectives; a recognition of achievements; and an identification of personal development needs ${ }^{108}$.

\section{Assessment}

The total range of written, oral and practical tests, as well as projects and $\rightarrow$ portfolios, used to decide on the learner's progress in the $\rightarrow$ course unit or in a module. These measures may be mainly used by the $\rightarrow$ learner to assess his/her own progress (formative assessment) or by the $\rightarrow$ teacher responsible to judge whether the course unit or module has been completed satisfactorily against the $\rightarrow$ learning outcomes of the unit or module (summative assessment).

\section{Assessment criteria}

Descriptions of what the $\rightarrow$ learner is expected to do, in order to demonstrate that a $\rightarrow$ learning outcome has been achieved.

\section{Audit}

A planned and documented activity performed by qualified personnel to determine by investigation, examination, or evaluation of objective evidence, the adequacy and compliance with established procedures, or applicable documents, and the effectiveness of implementation. Audit in healthcare is a process used by health professionals to assess, evaluate and improve care of patients in a systematic way, from simple data collection e.g. in medical records to the more complex sequence of steps entailed in completing the full-cycle (initial audit, change implemented, re-audit to demonstrate improvement). Audit measures current practice against defined (desired) standard and explicit criteria. It forms part of clinical governance, which aims to safeguard a high quality of clinical care for patients. The key component of clinical audit is that $\rightarrow$ performance is reviewed (or audited) to ensure that what should be done is being done, and if not it provides a framework to enable improvements to be made.

\section{Blueprint}

Bringing the relative importance of different clinical areas, covered in an $\rightarrow$ assessment procedure in accordance with the large variety of cases and problems 
and their prevalence in real practice. In the broad range of GP problems, blueprinting is important, because of the problem of $\rightarrow$ case specificity.

\section{Case based discussion (CBD)}

Structured oral interview across a range of competency areas; the starting point is the written record of cases selected by the trainee ${ }^{109}$.

\section{Case specifity}

Research has shown that learning in medicine is very much case-specific. Mastering a limited set of cases and/or problems does not guarantee the mastering of other cases, areas and problems, especially in a large field like GP/FM. So $\rightarrow$ assessment based on the handling of one or a few cases only gives a very restricted information on the $\rightarrow$ competence of a candidate. Assessment with 10 small tasks of five minutes selected with a good $\rightarrow$ blueprint generally gives much more valid information than one long case of 50 minutes.

\section{Checklist}

A list of $\rightarrow$ competencies to be mastered at the end of a training period, formatted as a clear defined list, checkable by the $\rightarrow$ learner, by the $\rightarrow$ teacher or by both, providing a constant overview of what is already mastered and what is still to be learned.

\section{Consultation observation tools (COT)}

Tools to assess consultation skills, can be used to assess video recorded consultations or during direct observation in general practice settings ${ }^{110}$.

\section{Essay method}

Written $\rightarrow$ reflection on specific questions, in the extended response kind (describe what should be done for...) or the restricted response kind (given this statement, describe this specific issue). Problem is the time needed and the low $\rightarrow$ reliability.

\section{Feedback}

e. g. by patients, relatives of patients, or staff. There is now convincing evidence that systematic feedback delivered by a credible source can change clinical $\rightarrow$ performance, although there are many complexities that influence the effectiveness of feedback in practice ${ }^{111,112,113}(\rightarrow$ multi-source feedback). 


\section{MCQ-MEQ method}

Multiple Choice Questionnaire: a format of objective measurement of the $\rightarrow$ knowledge of the $\rightarrow$ learner. Later adapted to other formats: Modified Essay Questionnaire, the Extended Matching type, etc. MEQ includes clinical reasoning, not only knowledge testing.

\section{Multi-source feedback}

A unique form of $\rightarrow$ workplace-based assessment in that it uses a collection of untrained raters, and the $\rightarrow$ feedback based on the collated ratings is subsequently fed back to the trainee or doctor by the $\rightarrow$ supervisor or peer. Thus it has aspects of $\rightarrow$ assessment of and for learning.

\section{Objective test method}

Includes a wide variety of test formats, in which the marking or the answer is objective: true/false questions; $\rightarrow$ Multiple Choice Questions; context-dependent questions, where a degree of analysis is needed to find the answers; extended matching questions, i. e. more complex combination of themes, scenarios, wide range of possible options, sometimes in relation to specific conditions.

\section{Observation method, direct}

Direct $\rightarrow$ observation of $\rightarrow$ performance on technical or interpersonal skills in the real, simulated or examination setting: sit-in with real patient in clinical practice or $\rightarrow$ simulated patients (SOO, simulated office oral); video-taped consultation with real patients in clinical practice or simulated patients with standardised assessment ${ }^{114}$. Observation by oneself, $\rightarrow$ tutor/ $\rightarrow$ supervisor, peers, etc. $\rightarrow$ Valid method, but $\rightarrow$ reliability is low. It can be made more objective by the use of $\rightarrow$ checklists, rating forms, and training the examiners.

\section{Observation method, indirect}

Simulates direct observation e.g. by using patient records (chart $\rightarrow$ audit), medical certificates, progress reports or by using patient-case discussion.

\section{On-site peer assessment}

$\rightarrow$ workplace-based assessment, $\rightarrow$ peer (group) assessment

\section{Oral method}

Traditionally the most used method, with a high face $\rightarrow$ validity, but very time consuming and unreliable. Remedies are standardisation of the content (by clear 
definition, by selection of a standard set, by using $\rightarrow$ standardised patients etc), or reducing examiners inconsistency (rating sheets, multiplying examiners with independent marking).

\section{OSCE method}

Objective Structured Clinical Examination: a format of objective evaluation, focused on (complex) skills testing through lists of wanted and unwanted features in relation to the skill.

\section{Patient satisfaction questionnaire}

Provides patient $\rightarrow$ feedback on doctors' empathy and relationship-building skills during consultations ${ }^{115}$.

Peer (group) assessment

$\rightarrow$ Assessment is done by peers and not by $\rightarrow$ tutors/ $\rightarrow$ supervisors. Different formats can be used. One specific is the 360 degree assessment format, where at least 10 colleagues, health personnel and staff contribute to the assessment.

\section{Portfolio, educational or reflective}

A portfolio is a summary of the major teaching activities and accomplishments, in relation with a Curriculum Vitae, including products and publications. It becomes a reflective or educational portfolio by adding a reflective part, where the $\rightarrow$ learner reflects on the personal learning process ${ }^{116}$. It can be used in a printed or in an electronic version.

\section{Reliability}

Refers to the reproducibility of the scores on the $\rightarrow$ assessment; high score reliability indicates that if the test were to be repeated over time, examinees would receive about the same scores on retesting as they received the first time. Unless assessment scores are reliable and reproducible (as in an experiment) it is nearly impossible to interpret the meaning of those scores - thus, $\rightarrow$ validity evidence is lacking.

\section{Review}

e.g. of medical records ( $\rightarrow$ audit)

\section{Review of patient records (RPR)}

Review of the collection of documents that provides an account of each episode in which a patient visited or sought treatment and received care or a referral for care from a health care facility. 


\section{Self assessment method}

Evaluation method to help learner's understanding of own ability and $\rightarrow$ performance. Criteria and standards are defined in a series of small group meetings by staff and $\rightarrow$ learners. Then learners use the criteria to judge their own performance.

\section{Short answer method}

Judging by asking specific short answers on given clinical vignettes: what is the diagnosis, list two typical symptoms, etc.

\section{Simulated patient}

Simulated patient (SP), or standardized patient (SP) (also known as a patient instructor) in health care is a healthy subject, or an actual patient who has been trained to portray accurately and consistently a particular patient case in order to simulate a set of symptoms or problems, and who is also trained to assess the $\rightarrow$ performance of students, trainees or doctors based on pre-defined criteria ${ }^{117}$.

\section{Structured written answer method}

After a given variable amount of patient data follows a series of options, between which the learner has to select the requested answer. Two types: the Patient Management Problem and the $\rightarrow$ Modified Essay Questionnaire on a broader field of possible options.

\section{Validity}

Refers to the evidence presented to support or refute the meaning or interpretation assigned to $\rightarrow$ assessment results. All assessments require validity evidence and nearly all topics in assessment involve validity in some way. Validity is the sine qua non of assessment, as without evidence of validity, assessments in medical education have little or no intrinsic meaning.

\section{Video observation}

\section{$\rightarrow$ observation method, direct}

\section{Workplace-based assessment (WPBA)}

WPBA is the $\rightarrow$ assessment of $\rightarrow$ competence and $\rightarrow$ performance based on what a trainee or doctor actually does in the workplace. The main aim of WPBA is to aid learning and reflecting (assessment for learning) by providing trainees and doctors with constructive $\rightarrow$ feedback and to support development. Train- 
ees and doctors can use the same methodology to assess themselves (reflective practice). The assessments help the $\rightarrow$ supervisors, $\rightarrow$ tutors and peers to chart a trainee's progress during a placement or a doctor's performance in daily practice. One major advantage of workplace-based assessment is its ability to evaluate performance in context ${ }^{118,119,120,121,122,123,124,125,126}$ ( $\rightarrow$ on-site peer assessment). Saturation can be sought by applying a variety of assessment methods, by 360 degree assessment ( $\rightarrow$ peer (group) assessment) and/or by longitudinal assessment ( $\rightarrow$ multi-source feedback). 
